\title{
Tuning the Gelation of Thermoresponsive Gels
}

\author{
Anna P. Constantinou and Theoni K. Georgiou* \\ Department of Materials \\ Imperial College London \\ Exhibition Road \\ London, SW7 2AZ \\ United Kingdom
}

European Polymer Journal

January 2016

*corresponding author 


\begin{abstract}
Thermoresponsive gels are exciting polymeric materials with many biomedical applications in medical devices, drug delivery, tissue engineering and bio-printing. Also, they have great potential to be used in 3-D printing and thus in the fabrication of many different devices and materials. As it is crucial for the application of these gels to be able to control and tailor the gelation temperature and concentration this was the main focus and point of discussion of this feature article. Thus, it is discussed in detail how by varying the molar mass, composition, stereochemistry and architecture the thermoresponsive properties of these gels are altered.
\end{abstract}

Keywords: physical gels, hydrogels, thermoresponsive polymers, temperature responsive polymers, thermoresponsive gels, block copolymers, triblock copolymers, injectable gels, gelation temperature, critical gelation concentration. 


\section{Abbreviations}

AA Acrylic acid

Bipolymer A polymer that is based on two different monomers.

DL-Ala D,L-Alanine

L-Ala L-Alanine

BO Butylene oxide

BMSiVE 2-(tert-Butyldimethylsilyloxy)ethyl vinyl ether

BuMA n-Butyl methacrylate

CGC Critical gelation concentration

CL Caprolactone

Copolymer A polymer that is based on more than one monomers.

$\boldsymbol{D} \quad$ Dispersity (see MMD)

DAAc 2-Carboxyethyl acrylate (DAA)

3-D 3-Dimensional

DMA $\quad N, N$-Dimethylacrylamide

DMAEA 2-(Dimethylamino)ethyl acrylate

DMAEMA 2-(Dimethylamino)ethyl methacrylate

EOEOVE 2-(2-Ethoxy)ethoxyethyl vinyl ether

EA Ethylene adipate

EG Ethylene glycol

EO Ethylene oxide (same as EG)

ESc Ethylene succinate

EtMA Ethyl methacrylate

GA Glycolide

GTP Group transfer polymerisation

HA Hexamethylene adipate

HMA n-Hexyl methacrylate

HEMA 2-Hydroxyethylmethacrylate

NIPAm N-isopropylacrylamide

LA Lactide

DLLA D,L-Lactide

LLA L-Lactide

LCST Lower critical solution temperature

MM Molar mass (same as molecular mass) 
MMD Molar mass distribution

MOVE 2-methoxyethyl vinyl ether

MPEG Methoxypoly(ethylene glycol)

PEG Poly(ethylene glycol)

PEGMA Poly(ethylene glycol) methyl ether methacrylate

PDMAEMA Poly[(2-dimethylamino)ethyl methacrylate]

PNIPAAm Poly( $N$-isopropylacrylamide)

PO Propylene oxide)

PVME Poly(vinyl methyl ether)

SO Styrene oxide

$\boldsymbol{T}_{\mathrm{g}} \quad$ Glass transition temperature

$\boldsymbol{T}_{\text {gel }} \quad$ Gelation temperature

Terpolymer A polymer that is based on three different monomers.

TRG Thermoresponsive gels 


\section{Introduction}

Thermoresponsive polymers are polymers that exhibit a drastic change in their physical properties in response to temperature and hence they are also called temperatureresponsive polymers. In solution this discontinuous change in physical properties manifests as a change in solubility and with a change in temperature the polymer becomes insoluble and precipitates out from solution. Polymers that become hydrophobic when increasing temperature demonstrate a Lower Critical Solution Temperature (LCST) behaviour that can be clearly observed visually since the solution becomes cloudy. The phase diagram of this behaviour is shown in Fig 1 (a). Each of the points on the curve is a cloud point while the minimum of the curve corresponds to the LCST. The most common thermoresponsive polymers that exhibit LCST behaviour are poly( $N$-isopropylacrylamide) (PNIPAAm) ${ }^{1-4}$ poly[2-(dimethylamino)ethyl methacrylate] (PDMAEMA), ${ }^{5}$ poly(vinyl methyl ether) (PVME), ${ }^{1}$ and poly(ethylene glycol) (PEG). ${ }^{6,7}$ The chemical structures of their repeating units are shown in Fig. 2. However, one has to be careful when quoting a transition temperature (the LCST or a cloud point) as this is influenced not just by the solvent (or solvent mixtures) and the ionic strength of the solution, but also by the molar mass (MM) and the molar mass distribution (MMD, dispersity, $Ð$ ) of the polymer. ${ }^{4-7}$ In certain conditions and usually when the thermoresponsive polymers are combined with other comonomers their aqueous solutions can form physical gels when increasing the temperature. A physical gel is a 3-dimensional (3-D) polymer network that is held together via physical entanglements, hydrogen bonds and/or hydrophobic interactions. ${ }^{8,9}$ The phase diagram of these aqueous based systems of thermoresponsive gels is shown in Fig 1(b).

This solution-gel (sol-gel) transition is thermo-reversible and thus these gels are also often called thermally-reversible gels as well as thermoresponsive gels (TRGs). This transition can be observed visually via the formation of a gel (the solution no longer flows, see Fig. 3) and 
phase diagrams like the one shown in Fig 1(b) can be constructed where the points on the green curve are gelation points. The gelation point can more accurately be determined via rheological measurements and it is taken as the instant when elastic modulus, G'exceeds the viscous modulus $\mathrm{G}^{\prime \prime}$ (i.e. the solid phase becomes dominant) ${ }^{10}$ as shown in Fig 3 . When increasing the temperature syneresis is often observed where liquid is also present with the gel, a phenomenon that was first reported by Graham in 1864 when observing gelatine solutions. $^{11}$

TRGs have many biomedical applications ${ }^{12}$ including drug delivery ${ }^{13-21}$ and tissue engineering as in-situ forming gels ${ }^{22-27}$ and in 3-D bioprinting. ${ }^{28-31}$ Furthermore with the increasing recent interest in 3-D printable materials and the need to control the rheology of the solution while printing ${ }^{32,33}$ the areas of potential usage of TRGs has increased since 3-D printing is applied to the manufacture of materials in various industries besides the medical industry, like aerospace, automotive, building and construction, marine, food industry and in manufacturing electronic and optical devices. ${ }^{34-36}$

In TRGs applications it is highly important to be able to control the gelation point, i.e. the temperature that the polymer solution becomes a gel, $T_{\text {gel }}$. Ideally this transition should be clear and sharp and not be happening over a range of temperatures. The latter can be improved by using well-defined polymers of narrow MMD. Since the control and tailorability of the gelation temperature is crucial it is also the focus of this feature article and specifically how the polymer characteristics affect the gelation. Thus, the main factors such as MM, composition, stereochemistry, architecture and topology of the polymer affecting the gelation of the polymer solutions will be identified and discussed. At this point it is important to note that only systematic studies will be discussed where one of these parameters has been varied independently, so clear conclusions can be drawn. The main chemical structures of the polymers and their repeating units that are being reviewed are shown in Fig 2 and the main 
parameters are highlighted in Table 1. Note that not all polymer structures shown are thermoresponsive but they have been combined with other thermoresponsive repeating units to produce thermoresponsive polymers.

\section{Effect of Molar Mass}

It is well documented that by increasing the MM of a polymer the viscosity of the polymer solution will increase since this follows the Mark-Houwink equation. In terms of

TRGs, when increasing the MM of the polymer, normally the gelation temperature $\left(T_{\text {gel }}\right)^{37-42}$ and/or the concentration that the gel is obtained at (critical gelation concentration, CGC) ${ }^{37,38}$ is decreased. The earliest systematic study where it was observed that both the $T_{\text {gel }}$ and CGC decreased when increasing the MM was carried out by Vadnere et al. in 1984 on Pluronics® with MMs ranging from 8500 to $13000 \mathrm{~g} \mathrm{~mol}^{-1} .{ }^{43}$ Later studies with similar observations on Pluronics ${ }^{\circledR}$ with smaller MM from 3400 to $6500 \mathrm{~g} \mathrm{~mol}^{-1}$ were also reported. ${ }^{38}$ Other studies on ABA triblock copolymers of different chemical structures than Pluronics®, specifically based on ethylene oxide (EO, blocks A) and butylene oxide (BO, block B) instead of propylene oxide (PO) with MM ranging from 6400 to $15500 \mathrm{~g} \mathrm{~mol}^{-1}$ found that $T_{\text {gel }}$ as well as CGC decreased by increasing the MM. ${ }^{37}$

In another study, three pairs of random copolymers based on (i) $\mathrm{N}$-isopropyl acrylamide (NIPAAm) and acrylic acid (AA), (ii) NIPAAm and 2-(dimethylamino)ethyl acrylate (DMAEA) and (iii) NIPAAm and 2-carboxyethyl acrylate (DAA) of different MMs (ranging from 300 to $500 \mathrm{~kg} \mathrm{~mol}^{-1}$ ), were synthesised via free radical polymerisation. ${ }^{39}$ Similarly, it was demonstrated that by increasing the MM the $T_{\text {gel }}$ decreased. ${ }^{39}$

In a study by Tarasevich et al. the $T_{\text {gel }}$ decreased and the gel strength increased while increasing the MM (5300 to $6400 \mathrm{~g} \mathrm{~mol}^{-1}$ ) of graft polymers where the backbone consisted of D,L-lactide (DLLA) and glycolide (GA), and the side chains consisted of EO. ${ }^{40}$ 
In a contradictory study on amphiphilic thermoresponsive poly $(N$-substituted $\alpha / \beta$ asparagine) derivatives, where polycondensation was used to prepare the polymers, with MMs ranging from 1100 to $5400 \mathrm{~g} \mathrm{~mol}^{-1}$ it was observed that the polymer with the lowest MM did not demonstrate thermoresponsive behaviour while the rest of the polymers demonstrated similar $T_{\text {gel }} \mathrm{s} .{ }^{44}$ However this observation is ambiguous as the polymers were synthesised with polycondensation and they had a very broad MMD.

In an interesting study on two multiblock copolymers based on NIPAm and $N, N$ dimethylacrylamide (DMA) with the same composition but two different MM, 46 and $95 \mathrm{~kg}$ $\mathrm{mol}^{-1}$ it was observed that only the higher MM polymer was able to form a gel while the smaller MM polymer underwent phase separation. ${ }^{41}$ Their difference was explained in terms of the micellar size. Regarding the higher MM polymer, at high temperatures, the NIPAAm blocks formed unimolecular micelles in the nanometer scale, bridged by the DMA blocks, leading to gel formation. The shorter one formed multimolecular micelles that were of the order of a hundred times larger than the latter. Thus, the short DMA blocks were hidden in those structures and were unable to contribute to the bridging, leading to phase separation. ${ }^{41}$

In a more recent study by our group, three families of $\mathrm{ABC}$ triblock copolymers were synthesised via group transfer polymerisation (GTP). ${ }^{42} \mathrm{~A}, \mathrm{~B}$ and $\mathrm{C}$ blocks were based on methoxy poly(ethylene glycol) methacrylate (PEGMA), $n$-butyl methacrylate (BuMA) and 2(dimethylamino)ethyl methacrylate (DMAEMA), respectively. Each family consisted of three copolymers having MM equal to 5000, 10000 and $15000 \mathrm{~g} \mathrm{~mol}^{-1}$, respectively. The families differed in the composition and this effect will be discussed in the following section. The family of the polymers with the lowest hydrophobic content did not form a gel at any concentration or temperature tested. For polymers with intermediate hydrophobic content the $T_{\mathrm{gel}}$ decreased as the MM increased with the highest MM polymers forming a gel at all 
temperatures tested. Similar results were observed for the polymers with the highest hydrophobic content with the two highest MM polymers forming a gel at all temperatures. ${ }^{42}$

Thus, it can be clearly concluded that by increasing the MM both CGC and $T_{\text {gel }}$ decrease, so if a clear sol-gel transition is desirable, both too low and too high MMs should be avoided and an optimal intermediate MM should be identified for the given system (of constant chemistry, composition and architecture). Finally, increasing the MM also enhances the mechanical properties and this can be crucial for some applications.

\section{Effect of Composition/ Hydrophobicity}

The effect of composition is more dominant when the content that is varied is the hydrophobic content. Specifically, a clear trend is observed where both the CGC and $T_{\text {gel }}$ decrease by increasing the hydrophobic content of the polymer while keeping the MM, the architecture and the chemistry of the polymer constant.

Our group reported two studies on $\mathrm{ABC}$ triblock copolymers where $\mathrm{A}, \mathrm{B}$ and $\mathrm{C}$ correspond to PEGMA, BuMA and DMAEMA, respectively, where the $T_{\text {gel }}{ }^{42,45}$ and CGC $^{45}$ decreased while the mechanical properties increased by increasing the hydrophobic BuMA content with the terpolymers with the lowest hydrophobic content not demonstrating a gelation at any temperatures or only if the polymer concentration was increased. In our more recent study the effect of the PEG length was examined as well as the hydrophobic content and it was observed that only polymers with high hydrophobic content and shorter PEG lengths form TRGs. ${ }^{46}$

In an additional systematic study by our group, four families having different architectures (statistical, ABA symmetric and two ABA asymmetric triblock copolymers) were synthesised via GTP and the composition was varied for each. ${ }^{47}$ The B and A blocks 
were based on DMAEMA and BuMA units, respectively. In all four families, an increase in composition of the hydrophobic BuMA decreased the $T_{\text {gel }}$ which is consistent with the previous observations.

In another study by Yu et al. on ABA copolymers where the A block was based on EO and the central B block consisted of both DLLA and GA of varying compositions the CGC was reduced as the content of the hydrophobic DLLA was increased. ${ }^{48}$ Graft polymers based on the same monomers were also investigated. Specifically DLLA and GA were on the backbone of the polymer while the EO units were side chains. By increasing the content of the hydrophobic DLLA the $T_{\text {gel }}$ decreased and an increase in the gel strength was observed. ${ }^{40}$

Chen et al. in a similar study to Yu et al. synthesised ABA copolymers but the composition of the A block was varied which was based on GA and L-lactide (LLA) while the B block was based on EO. ${ }^{49}$ However the $T_{\text {gel }}$ increased upon increasing the LLA/GA ratio while the CGC remained at similar values. ${ }^{49}$ This contradictory result regarding the $T_{\text {gel }}$ was probably due to the significant influence of the stereochemistry that counteracted the hydrophobic content effect as will be discussed later on. In another study on ABA triblock copolymers where the A blocks were based on both caprolactone (CL) and GA, of different ratios, whereas the B block consisted, as in the previous study, ${ }^{48}$ of EO ${ }^{50}$ Upon decreasing the CL/GA ratio, the $T_{\text {gel }}$ decreased; as expected since $\mathrm{CL}$ is hydrophobic and also has the ability to crystallize.

In Cohn et al. three different multiblock copolymers based on EO and PO with varying compositions were investigated and it was clearly demonstrated that increasing the hydrophobic PO content the $T_{\text {gel }}$ decreased. ${ }^{51}$

Kang et al. investigated diblock copolymers, where the first block was based on methoxy poly(ethylene glycol) (MPEG) and the second block on both CL and LLA units, 
randomly distributed. ${ }^{52}$ The CL/LLA ratio was varied and only polymers with a high ratio formed gels of which the $T_{\text {gel }}$ decreased with increasing CL content. This was attributed to the enhanced crystallinity of the polymer when increasing the CL content. In a complementary study by the same group 6 families of differing CL/LLA ratios were synthesised and in some cases, pendant groups were attached. ${ }^{53}$ These pendant groups were attached on the LLA units and they were either 3-benzyloxymethyl lactide (BnLA) units or their hydroxyl and carboxyl derivatives and they observed that the introduction of side groups disturbs the gelation possibly because it prevents the CL crystallinity thus the $T_{\text {gel }}$ increased compared with the non-modified polymers. Interestingly, when comparing the hydrophobicity/hydrophilicity of the pendant groups between them the more hydrophobic were able to affect the CL crystallinity more and thus the polymers with hydrophobic side groups presented higher $T_{\mathrm{gel}} \mathrm{S}$ than the ones with hydrophilic side groups. ${ }^{53}$

In all the following studies the hydrophobicity was varied by not changing the ratio of the hydrophobic/hydrophilic units but by fabricating different polymers where the thermoresponsive units were kept the same but the comonomer was changed.

Our group synthesised 4 families of terpolymers of different architectures ABC, ACB and BAC triblock copolymers and the corresponding statistical copolymers and for each of those families the length of the side alkyl group was varied. ${ }^{54}$ Specifically A and C were based on PEGMA and DMAEMA, respectively and were always kept the same while the B monomer was varied, specifically; ethyl methacrylate (EtMA), BuMA and $n$-hexyl methacrylate (HMA) were used. For all architectures the viscosity increased by increasing the length of the hydrophobic monomer and where a sol-gel transition was observed the $T_{\text {gel }}$ decreased by increasing the length of the side group. Interestingly the stability of the gel formed was reduced by increasing the alkyl side group. This was attributed to the 3 hydrophobic monomers having different glass transition temperatures $\left(T_{\mathrm{g}} \mathrm{s}\right)$. The shorter the 
alkyl side group, the higher the $T_{\mathrm{g}}$, thus meaning the more 'glassy' was the hydrophobic micellar core. Therefore, the elasticity increased upon increasing the hydrophobic alkyl side chain, leading to reduced stability of the gels. ${ }^{54}$

Two studies on ABA triblock copolymers were carried out. ${ }^{55,56}$ In the first one the A blocks consisted of EO and the hydrophobicity of the B block was varied by using hexamethylene adipate (HA), ethylene adipate (EA) or ethylene succinate (ESc) units. By decreasing the hydrophobicity of the B block the CGC decreased. ${ }^{55}$ In the second study a Pluronic ${ }^{\circledR}$ was compared with two copolymers having instead of PO units, DLLA and both DLLA and GA, respectively. ${ }^{56}$ Similarly with the first study the CGC decreased upon increasing the hydrophobicity and the $T_{\text {gel }}$ also decreased. ${ }^{56}$

Overall, increasing the hydrophobicity of the polymers usually decreases the CGC ${ }^{45}$, $46,48,55,56$ as well as the $T_{\text {gel }} 40,42,45-47,49-52,54,56$ unless hydrophobic groups are introduced that disturb the aggregation/crystallisation of another hydrophobic component. ${ }^{53}$ Again an optimal hydrophobic/hydrophilic ratio is needed in order to have a clear sol-gel transition since high ratios cause gelation at all temperatures and/or phase separation of the polymer from the aqueous based solutions.

\section{Effect of Stereochemistry}

Some of the monomers used to produce thermoresponsive polymers contained chiral centres (see Fig 2); thus leading to polymers of varying stereochemistry. In the cases where those monomers were incorporated in the thermoresponsive polymer backbone, their behaviour and their properties were affected. Therefore, in this section, the attention is focused on the effect of stereochemistry. 
In Joo et al. , two multiblock copolymers consisting of EO and DLLA or LLA units, respectively, were synthesised. ${ }^{57}$ The thermoresponsive behaviour was studied by visual and rheological tests. A sol-gel transition, followed by syneresis, was observed; regardless of the stereochemistry. However, concerning the LLA based copolymer the first and second phase transitions were shifted to lower and higher temperatures, respectively; leading to a wider gel window. In addition, the CGC decreased and maximal gel modulus increased after changing from DLLA to LLA. The LLA based segments, characterised by isotacticity, enhanced the hydrophobic interactions and consequently the aggregation. Also, the increased gel modulus in the LLA based copolymer was attributed to the reduced motion of the methyl groups. ${ }^{57}$

Two studies on ABA triblocks were reported. ${ }^{49,58}$ In the first study, two series of polymers were investigated where the B block was kept constant; EO based, while the A block was varied by being based either on GA and LLA or GA and DLLA. ${ }^{49}$ The copolymers of each series possessed different compositions; discussed previously. For the polymers with higher lactide (LA)/GA ratio, the $T_{\text {gel }}$ of the DLLA based copolymer was lower than that of the LLA based one; determined after visual tests. Interestingly, the shift of $T_{\text {gel }}$ to higher values, by changing from DLLA to LLA, led to the $T_{\text {gel }}$ of the latter at body temperature. This was attributed to the steric isotacticity of the LLA based copolymer. Concerning the ones with the lower LA/GA ratio, similar gelation temperature, maximal gel modulus and sticky morphology were observed; due to the low content in chiral centres. ${ }^{49}$ In the second study ABA triblocks copolymers with A and B based on LLA or DLLA and EO, respectively, were studied by visual and rheological tests. ${ }^{58}$ According to visual tests, above a certain concentration, the LLA and DLLA based copolymers did and did not gel, respectively. After rheological measurements, the stiffness of the gel reduced with the same order. The crystallinity and the amorphous structure of the of LLA and DLLA based, respectively, were found to be responsible for their differences. ${ }^{58}$ 
In summary, it is not easy to predict how the stereochemistry affects the gelation as this is also associated with the polymer composition but it should be kept in mind that the stereochemistry of the polymer does have an impact and can really influence the gelation and the properties of the gel.

\section{Effect of Architecture and Topology}

The position of the monomers or/and the blocks within the polymer chain also influences the thermoresponsive behaviour of the TRGs and in some cases the properties of the resulting gels.

\section{Random vs Gradient vs Block Structure}

Whether the sequence of the repeating units is in a random, a gradient or a block structure has a significant effect on the gel formation and the properties of the gel. Specifically in many occasions where random and block copolymers were compared the random polymer either did not form a gel ${ }^{47,54,59-61}$ or formed a less stable gel at higher $T_{\text {gel. }}{ }^{45}$, ${ }^{54}$ This effect was observed for both bipolymers ${ }^{59-61}$ and terpolymers ${ }^{45,47,54}$ (two and three component based polymers, respectively). For bipolymers these were observed for diblock copolymers based on 2-(2-ethoxy)ethoxyethyl vinyl ether (EOEOVE) and 2-(tertbutyldimethylsilyloxy)ethyl vinyl ether (BMSiVE) ${ }^{59}$ and EOEOVE and 2-methoxyethyl vinyl ether (MOVE). ${ }^{60,61}$ The last study on EOEOVE and 2-methoxyethyl vinyl ether (MOVE) also included a gradient polymer that was able to form gels but not in such a wide temperature window as the block copolymer. ${ }^{61}$ The only study on triblock bipolymers involved ABA triblock copolymers where the A and B blocks were based on BuMA and DMAEMA, respectively, and the observation was similar; the block structure forms gels more easily than the random structure. ${ }^{54}$ All the studies on terpolymers were performed by our group and were based on PEGMA ${ }^{45,54}$ (A block), DMAEMA (C block) ${ }^{45,54}$ and either 
EtMA,${ }^{54}$ BuMA $^{45,54}$ or HMA $^{54}$ (B block) and they are in agreement with previous observations. When the random copolymers did form a gel it was either because the MM was relatively high ${ }^{45}$ or because the most hydrophobic, $\mathrm{HMA}^{54}$ comonomer was used.

In a more complex study ABA triblock copolymers were made where the B block was based on EO units and the A block was based on both DLLA and GA and their sequence was varied. ${ }^{62}$ Specifically, three copolymers were synthesised that differed in the sequence of the DLLA and GA, from a more random to a more gradient structure, and the $T_{\text {gel }}$ decreased as the structure was less random. ${ }^{62}$

\section{Diblock vs Triblock Copolymers}

Two studies where AB diblock copolymers were compared to their corresponding ABA triblock copolymers were performed and in both it was found that the diblock copolymers formed gels easier at lower CGC. ${ }^{63,64}$ The polymers in the first study were based on EO and styrene oxide (SO), ${ }^{63}$ and in the second study on $\mathrm{EO}$ and $\mathrm{BO}^{64}$ with the hydrophobic monomers SO and BO being always the B block. The easiest gel formation by the diblock copolymers was attributed to more stable micelles formed by these due to the low micellisation enthalpy. ${ }^{63}$

In another study $\mathrm{AB}, \mathrm{ABA}$ as well as a $\mathrm{BAB}$ copolymer were compared, based on $\mathrm{EO}$ and $\mathrm{BO}$ and the results were similar to the above with the two triblocks not forming a gel while the $\mathrm{AB}$ diblock copolymer did. ${ }^{65}$ In a study by the same authors using the same monomers but by increasing the hydrophobic content gelation was achieved by the triblock copolymers too however the CGC increased from the diblock to the ABA triblock to the $\mathrm{BAB}$; indicating once again the easier gelation of the diblock compared to the corresponding triblock copolymers. ${ }^{66}$ 


\section{$A B A$ versus $B A B$ triblock copolymers}

It is not easy to compare the $\mathrm{ABA}$ versus $\mathrm{BAB}$ studies as they are ambiguous. Specifically Bae et al. compared two triblock bipolymers with the A and B block based on EO and CL, respectively and observed that the $T_{\text {gel }}$ for the BAB was lower than the ABA copolymer solutions. ${ }^{67}$ The difference was attributed to the different micellar structure. The core and the shell of the micelles formed in the ABA triblock copolymer consisted of the hydrophobic CL and the hydrophilic EO segments, respectively. In contrast, bridging between the micelles led to gelation in $\mathrm{BAB}$ triblock copolymer. These bridges were also responsible for the higher storage modulus of the $\mathrm{BAB}$ than the ABA triblock copolymer

However in a contradictory study where the A and B blocks consisted of NIPAAm and 2-hydroxyethylmethacrylate (HEMA) ${ }^{68}$ it was observed that the ABA had a lower $T_{\text {gel }}$ than the BAB counterpart but it should be pointed out that the ABA had solubility issues. Comparing the two opposing studies is not easy as the thermoresponsive components have quite different LCSTs as homopolymers (PEO much higher than PNIPAm) and also the CL based block will be able to crystallize and enhance aggregation unlike the HEMA based block. However we believe that the CL crystallinity is the dominant effect that changes the trend since the previous study on EO and BO agrees ${ }^{66}$ with the NIPAAm and HEMA study. ${ }^{68}$

\section{Asymmetry Effect}

In a more complex study by our group on symmetric and asymmetric ABA triblock copolymers based on BuMA and DMAEMA (as the A and B, blocks, respectively) the effect of the asymmetry of the A blocks was investigated. ${ }^{47}$ Specifically, the symmetric ABA triblock copolymers were compared with the ABA' and ABA'" with the same composition; where A' and A'" equal to $2 \mathrm{~A}$ and $4 \mathrm{~A}$, respectively and it was clearly observed that by increasing the asymmetry of the polymers the $T_{\text {gel }}$ increased. ${ }^{47}$ This was attributed to 
insufficient bridging of the flower-like micelles to form a gel when the polymers are less symmetric.

\section{Effect of 3-D structure (linear versus H-shaped copolymer)}

Only one systematic study was reported where polymers of different topologies were investigated and the architecture was varied independently. Specifically, $\mathrm{A}_{2} \mathrm{BA}_{2}$ block copolymers, named as H-shaped with four arms with linear ABA triblock copolymers. ${ }^{69}$ The A and B blocks were formed by NIPAAm and EO, respectively. Concerning the gelation temperature and the change of the viscosity upon altering the temperature, obtained after visual and rheological tests, respectively, no differences were observed. Therefore, it was concluded that, in this specific case, the change in the 3-D architecture did not influence the thermoresponsive properties of the copolymers. ${ }^{69}$

\section{Effect of the Sequence of the Blocks in Triblocks Copolymers (ABC vs ACB vs BAC)}

Only our group and Park et al. ${ }^{70}$ have studied triblock terpolymers with all three blocks being based on different monomers; usually ABA triblock terpolymers have been reported where one block was a statistical polymeric segment of two monomers.

We investigated in two studies how the position and sequence of the blocks affects the thermoresponsive properties i.e. compared $\mathrm{ABC}$ vs $\mathrm{ACB}$ vs BAC polymer architecture where A was based on PEGMA, B was the hydrophobic monomer (either EtMA, ${ }^{54}$ BuMA $^{45,54}$ or/and $\mathrm{HMA}^{54}$ ) and C was based on DMAEMA. Random terpolymers were also synthesised but as we have previously stated random copolymers either did not form gels or formed ones that were unstable and at higher $T_{\mathrm{gel}} \mathrm{S}$.

In terms of the triblock terpolymers, all of them were able to form gels with the BAC architecture able to form gels either at all temperatures ${ }^{45}$ or at lower $T_{\mathrm{gel}} \mathrm{S}^{54}$ compared to the 
other two triblock copolymer architectures. In this architecture (BAC) the thermoresponsive monomer with the lower LCST transition (DMAEMA) and the hydrophobic monomer (BuMA) form the outer blocks and it seems that the PEGMA block in the middle is not sufficient to prevent the enhanced aggregation of the micellar structures. However, it should be pointed out that these gels at higher temperatures destabilise easier than the gels formed by the $\mathrm{ABC}$ triblock copolymer where the hydrophobic block is in the middle. ${ }^{54}$ Moreover, the ABC triblock architecture was identified to be the "best" architecture because it was producing the most clear, sharp sol-gel transition and the gels seemed to remain more stable compared to the two other architectures.

Park et al. compared two triblock copolymer architectures, ABC and ACB, where A, B, and C consisted by EO, L-alanine (L-Ala), and D,L-alanine (DL-Ala). ${ }^{70}$ The thermoresponsive behaviour was investigated by rheological and visual tests. According to visual tests, the $\mathrm{ABC}$ polymer gelled upon increasing the temperature. The ACB formed a squeezed gel after the first sol-gel transition, which was not confirmed by rheology. The ABC also gelled at higher temperatures and concentrations than the ACB one. The difference was attributed to the different secondary structure at higher temperatures and the micellar shape. Specifically, the block consisted of L-Ala formed an $\alpha$-helix in both cases at low temperatures. Only in the case of the $\mathrm{ACB}$ one, upon increasing the temperature, was a random coil formed instead. ${ }^{70}$ Due to the effect of the stereochemistry a comparison of this study with the previous ones on $\mathrm{ABC}, \mathrm{BAC}$ and $\mathrm{ACB}$ is not feasible.

In summary, the polymer architecture has a significant effect on the thermoresponsive properties of the polymers and we have tried to demonstrate this effect in Figure 4. The thermoresponsive, hydrophilic (non-thermoresponsive) and hydrophobic components are coloured in green, blue and red, respectively. It can clearly be concluded that the block structure always promotes the formation of a gel when compared to random copolymers. 
When comparing $\mathrm{ABA}$ or $\mathrm{BAB}$ triblocks to the corresponding diblock the $\mathrm{AB}$ diblocks form gels more easily while the $\mathrm{ABA}$ versus $\mathrm{BAB}$ results were controversial but the majority of studies suggest that the $T_{\text {gel }}$ is lower for the ABA architecture. When comparing triblock terpolymers, with all blocks being based on three different components, when the hydrophobic block is in the middle the gel presents a clear sol-gel transition and the gel is more stable at higher temperatures. On the other hand, the architecture where the hydrophobic and the thermoresponsive monomers are the outer blocks, the polymers form gels at all temperatures or have a very low $T_{\text {gel }}$.

\section{Conclusions}

Several polymeric characteristics strongly influence the ability of thermoresponsive polymers to form gels. Specifically, by increasing the molar mass as well as the hydrophobic content of the polymer the ability of the polymer to form gels is enhanced. Stereochemistry also affects gelation but not in an easy to predict manner while the polymer architecture also has a significant effect. In particular, the block copolymer architecture is more efficient in forming gels when compared to the random copolymer architecture. Also diblock copolymers are more efficient in forming gels when compared to their ABA counterparts. When comparing triblocks copolymers, some studies are controversial, but overall having the hydrophobic and/or thermoresponsive blocks in the outer blocks seems to enhance gelation. However, it was the ABC triblock copolymers, where the B block was the hydrophobic structure that demonstrated a very clear sol-gel transition and the formation of a more stable gel at higher temperatures. Only one report studied a linear versus a non-linear polymer structure and found no effect but we believe more studies are required to fully investigate the effect of the 3-D structure on the TRG properties. We predict that the 3-D structure of the polymer will be an important factor in TRG properties since the noticeable difference in 
rheological properties of linear versus branched, star and graft polymers for example is wellestablished.

To conclude, we want to point out that forming the most stable or the stronger gel and/or reducing the CGC or the $T_{\text {gel }}$ is actually not always desirable for many of the applications of the TRGs. A clear and sharp sol-gel transition is required for example for the in-situ formation of scaffolds for tissue engineering. So, all these different polymer characteristics have to be controlled and tailored to obtain the optimal sol-gel transition and this can only be achieved by systematic studies where the parameters can be independently investigated and by fabricating well-defined polymers with narrow MMDs.

\section{Acknowledgements}

The Department of Materials at Imperial College London is thanked for funding APC. 


\section{References}

1. H. G. Schild and D. A. Tirrell, The Journal of Physical Chemistry, 1990, 94, 43524356.

2. X. Zhou, J. Li, C. Wu and B. Zheng, Macromolecular Rapid Communications, 2008, 29, 1363-1367.

3. C. Boutris, E. G. Chatzi and C. Kiparissides, Polymer, 1997, 38, 2567-2570.

4. A. Halperin, M. Kröger and F. M. Winnik, Angewandte Chemie - International Edition, 2015.

5. V. Bütün, S. P. Armes and N. C. Billingham, Polymer, 2001, 42, 5993-6008.

6. S. Saeki, N. Kuwahara, M. Nakata and M. Kaneko, Polymer, 1977, 18, 1027-1031.

7. S. Saeki, N. Kuwahara, M. Nakata and M. Kaneko, Polymer, 1976, 17, 685-689.

8. S. J. Buwalda, K. W. M. Boere, P. J. Dijkstra, J. Feijen, T. Vermonden and W. E. Hennink, Journal of Controlled Release, 2014, 190, 254-273.

9. $\quad$ L. Yu and J. Ding, Chemical Society Reviews, 2008, 37, 1473-1481.

10. G. E. Yu, H. Li, J. P. A. Fairclough, A. J. Ryan, N. McKeown, Z. Ali-Adib, C. Price and C. Booth, Langmuir, 1998, 14, 5782-5788.

11. M. Kunitz, The Journal of General Physiology, 1928, 12, 289-312.

12. M. A. Ward and T. K. Georgiou, Polymers, 2011, 3, 1215-1242.

13. D. P. Huynh, M. K. Nguyen, B. S. Pi, M. S. Kim, S. Y. Chae, K. C. Lee, B. S. Kim, S. W. Kim and D. S. Lee, Biomaterials, 2008, 29, 2527-2534.

14. K. Young Jin, S. Choi, K. Jae Joon, M. Lee, K. Kyung Soo and K. Sung Wan, Pharmaceutical Research, 2001, 18, 548-550.

15. G. M. Zentner, R. Rathi, C. Shih, J. C. McRea, M. H. Seo, H. Oh, B. G. Rhee, J. Mestecky, Z. Moldoveanu, M. Morgan and S. Weitman, Journal of Controlled Release, 2001, 72, 203-215. 
16. L. Yu, G. T. Chang, H. Zhang and J. D. Ding, International Journal of Pharmaceutics, 2008, 348, 95-106.

17. G. Chang, T. Ci, L. Yu and J. Ding, Journal of Controlled Release, 2011, 156, 21-31.

18. K. Li, L. Yu, X. Liu, C. Chen, Q. Chen and J. Ding, Biomaterials, 2013, 34, 28342842.

19. L. Yu, T. Ci, S. Zhou, W. Zeng and J. Ding, Biomaterials Science, 2013, 1, 411-420.

20. L. Yu, K. Li, X. Liu, C. Chen, Y. Bao, T. Ci, Q. Chen and J. Ding, Journal of Pharmaceutical Sciences, 2013, 102, 4140-4149.

21. T. Ci, L. Chen, L. Yu and J. Ding, Scientific Reports, 2014, 4.

22. E. S. Place, J. H. George, C. K. Williams and M. M. Stevens, Chemical Society Reviews, 2009, 38, 1139-1151.

23. S. P. L. Hoerstup, L.; Lysaght, M.J.; Mikos, A.G.; Rein, D.; Schoen, F.J.; Temenoff, J.S.; Tessmar, J.K.; Vacanti, J.P., Tissue engineering. In Biomaterials science: An introduction to materials in medicine, Eds. Elsevier Academic Press, San Diego, 2004.

24. L. Klouda and A. G. Mikos, Eur.J. Pharm. Biopharm., 2008, 68, 34-45.

25. J. D. Kretlow, L. Klouda and A. G. Mikos, Advanced Drug Delivery Reviews, 2007, 59, 263-273.

26. J. D. Kretlow, S. Young, L. Klouda, M. Wong and A. G. Mikos, Advanced Materials, 2009, 21, 3368-3393.

27. L. Klouda, European Journal of Pharmaceutics and Biopharmaceutics, 2015, 97, 338-349.

28. M. Kesti, M. Müller, J. Becher, M. Schnabelrauch, M. D’Este, D. Eglin and M. Zenobi-Wong, Acta Biomaterialia, 2015, 11, 162-172. 
29. M. Müller, J. Becher, M. Schnabelrauch and M. Zenobi-Wong, Journal of Visualized Experiments : JoVE, 2013, 50632.

30. R. Censi, W. Schuurman, J. Malda, G. di Dato, P. E. Burgisser, W. J. A. Dhert, C. F. van Nostrum, P. di Martino, T. Vermonden and W. E. Hennink, Advanced Functional Materials, 2011, 21, 1833-1842.

31. M. J. Sawkins, P. Mistry, B. N. Brown, K. M. Shakesheff, L. J. Bonassar and J. Yang, Biofabrication, 2015, 7.

32. M. Zhang, A. Vora, W. Han, R. J. Wojtecki, H. Maune, A. B. A. Le, L. E. Thompson, G. M. McClelland, F. Ribet, A. C. Engler and A. Nelson, Macromolecules, 2015.

33. J. K. Chen and C. J. Chang, Materials, 2014, 7, 805-875.

34. J.-Y. Lee, W. S. Tan, J. An, C. K. Chua, C. Y. Tang, A. G. Fane and T. H. Chong, Journal of Membrane Science, 2016, 499, 480-490.

35. W. Zhu, J. Ock, X. Ma, W. Li and S. Chen, in 3D Bioprinting and Nanotechnology in Tissue Engineering and Regenerative Medicine, eds. L. G. Zhang, J. P. Fisher and K. W. Leong, Academic Press, 2015, pp. 25-55.

36. P. Calvert, Chemistry of Materials, 2001, 13, 3299-3305.

37. C. V. Nicholas, Y.-Z. Luo, N.-J. Deng, D. Attwood, J. H. Collett, C. Price and C. Booth, Polymer, 1993, 34, 138-144.

38. K. H. Sun, Y. S. Sohn and B. Jeong, Biomacromolecules, 2006, 7, 2871-2877.

39. K. J. Krzyminski, M. Jasionowski and A. Gutowska, Polymer International, 2008, 57, 592-604.

40. B. J. Tarasevich, A. Gutowska, X. S. Li and B. M. Jeong, Journal of Biomedical Materials Research - Part A, 2009, 89, 248-254.

41. Z. Ge, Y. Zhou, Z. Tong and S. Liu, Langmuir, 2011, 27, 1143-1151.

42. M. A. Ward and T. K. Georgiou, Soft Matter, 2012, 8, 2737-2745. 
43. M. Vadnere, G. Amidon, S. Lindenbaum and J. L. Haslam, International Journal of Pharmaceutics, 1984, 22, 207-218.

44. Y. Takeuchi, T. Tsujimoto and H. Uyama, Polymers for Advanced Technologies, 2011, 22, 620-626.

45. M. A. Ward and T. K. Georgiou, Journal of Polymer Science, Part A: Polymer Chemistry, 2010, 48, 775-783.

46. A. Constantinou and T. K. Georgiou, Polymer Chemistry, 2016.

47. M. A. Ward and T. K. Georgiou, Journal of Polymer Science, Part A: Polymer Chemistry, 2013, 51, 2850-2859.

48. G. E. Yu, Z. Yang, M. Ameri, D. Attwood, J. H. Collett, C. Price and C. Booth, Journal of Physical Chemistry B, 1997, 101, 4394-4401.

49. C. Chen, L. Chen, L. Cao, W. Shen, L. Yu and J. Ding, RSC Advances, 2014, 4, 87898798.

50. L. Yu, W. Sheng, D. Yang and J. Ding, Macromolecular Research, 2013, 21, 207215.

51. D. Cohn, A. Sosnik and A. Levy, Biomaterials, 2003, 24, 3707-3714.

52. Y. M. Kang, S. H. Lee, J. Y. Lee, J. S. Son, B. S. Kim, B. Lee, H. J. Chun, B. H. Min, J. H. Kim and M. S. Kim, Biomaterials, 2010, 31, 2453-2460.

53. J. I. Kim, D. Y. Kim, D. Y. Kwon, H. J. Kang, J. H. Kim, B. H. Min and M. S. Kim, Biomaterials, 2012, 33, 2823-2834.

54. M. A. Ward and T. K. Georgiou, Polymer Chemistry, 2013, 4, 1893-1902.

55. M. J. Song, D. S. Lee, J. H. Ahn, D. J. Kim and S. C. Kim, Journal of Polymer Science, Part A: Polymer Chemistry, 2004, 42, 772-784.

56. M. J. Park and K. Char, Langmuir, 2004, 20, 2456-2465.

57. M. K. Joo, Y. S. Sohn and B. Jeong, Macromolecules, 2007, 40, 5111-5115. 
58. N. Sanabria-DeLong, S. K. Agrawal, S. R. Bhatia and G. N. Tew, Macromolecules, 2006, 39, 1308-1310.

59. S. Sugihara, K. Hashimoto, S. Okabe, M. Shibayama, S. Kanaoka and S. Aoshima, Macromolecules, 2004, 37, 336-343.

60. S. Sugihara, S. Kanaoka and S. Aoshima, Macromolecules, 2005, 38, 1919-1927.

61. K. I. Seno, I. Tsujimoto, T. Kikuchi, S. Kanaoka and S. Aoshima, Journal of Polymer Science, Part A: Polymer Chemistry, 2008, 46, 6151-6164.

62. L. Yu, Z. Zhang and J. Ding, Biomacromolecules, 2011, 12, 1290-1297.

63. S. Barbosa, M. A. Cheema, P. Taboada and V. Mosquera, Journal of Physical Chemistry B, 2007, 111, 10920-10928.

64. A. Kelarakis, V. Havredaki, L. Derici, G. E. Yu, C. Booth and I. W. Hamley, Journal of the Chemical Society - Faraday Transactions, 1998, 94, 3639-3647.

65. Z. Yang, S. Pickard, N.-J. Deng, R. J. Barlow, D. Attwood and C. Booth, Macromolecules, 1994, 27, 2371-2379.

66. Y. W. Yang, Z. Ali-Adib, N. B. McKeown, A. J. Ryan, D. Attwood and C. Booth, Langmuir, 1997, 13, 1860-1861.

67. S. J. Bae, J. M. Suh, Y. S. Sohn, Y. H. Bae, S. W. Kim and B. Jeong, Macromolecules, 2005, 38, 5260-5265.

68. X. Zhao, W. Liu, D. Chen, X. Lin and W. W. Lu, Macromolecular Chemistry and Physics, 2007, 208, 1773-1781.

69. M. Teodorescu, I. Negru, P. O. Stanescu, C. Drăghici, A. Lungu and A. Sârbu, Journal of Macromolecular Science, Part A: Pure and Applied Chemistry, 2011, 48, 177-185.

70. S. H. Park, B. G. Choi, H. J. Moon, S. H. Cho and B. Jeong, Soft Matter, 2011, 7, 6515-6521. 


\section{Figure Legends}

Figure 1: Phase diagram (a) of a thermoresponsive homo- and co-polymer that exhibits a LCST behaviour (b) a thermoresponsive copolymer that is able to form reversible gels.

Figure 2: Chemical structures and abbreviations of the main types of polymers used as well as their corresponding monomer units

Figure 3. Sol-gel transition as it is monitored by rheology. G' (elastic modulus), G". (viscous modulus) and complex viscosity are shown in solid, dotted and dashed lines, respectively. The gel point is defined as the temperature when elastic modulus, G'exceeds the viscous modulus $\mathrm{G}^{\prime \prime}$.

Figure 4: Schematic representations of the different polymer architectures. The thermoresponsive, hydrophilic and hydrophobic components are coloured in green, blue and red, respectively. With squares we emphasize which polymer structure forms gel easier. With dotted squares some discrepancies or interesting gel formations are noted. 

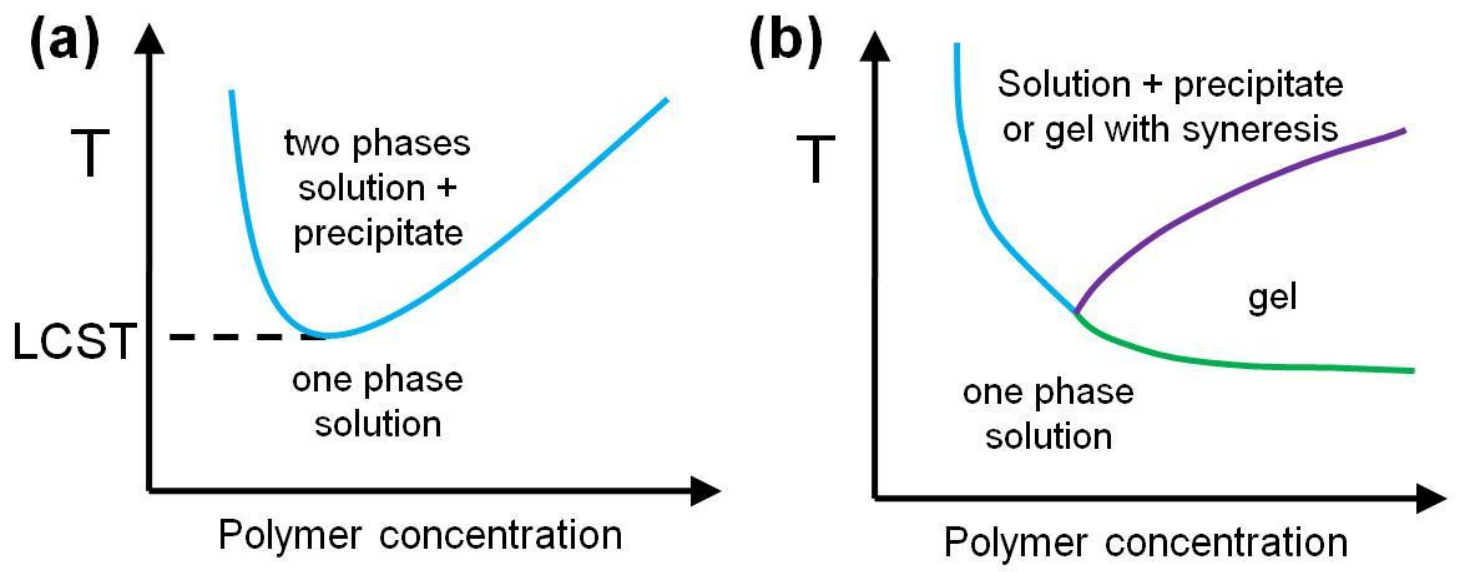

Figure 1: Phase diagram (a) of a thermoresponsive homo- and co-polymer that exhibits a LCST behaviour (b) a thermoresponsive copolymer that is able to form reversible gels. 


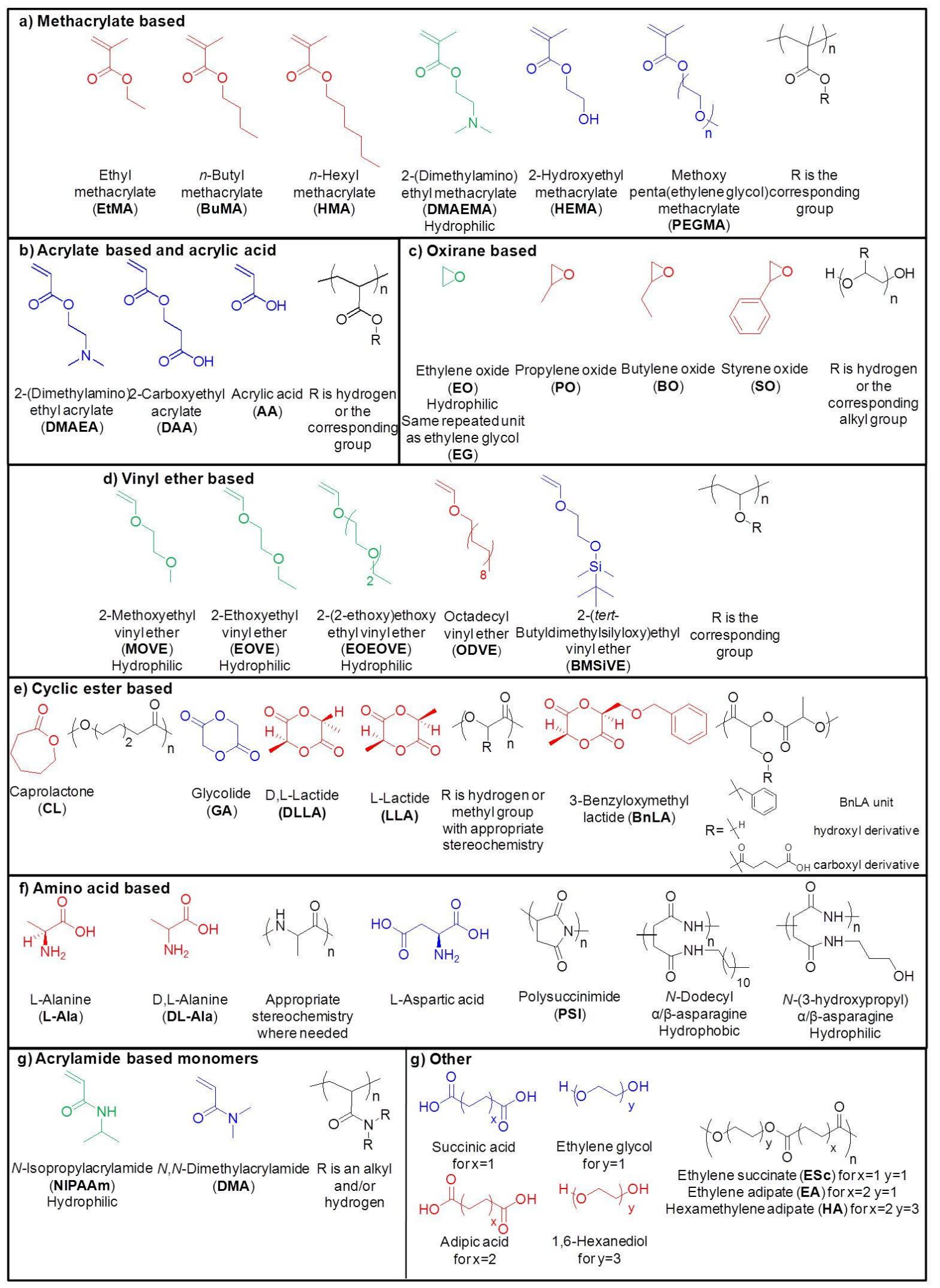

Figure 2: Chemical structures and abbreviations of the main types of polymers used as well as their corresponding monomer units. 


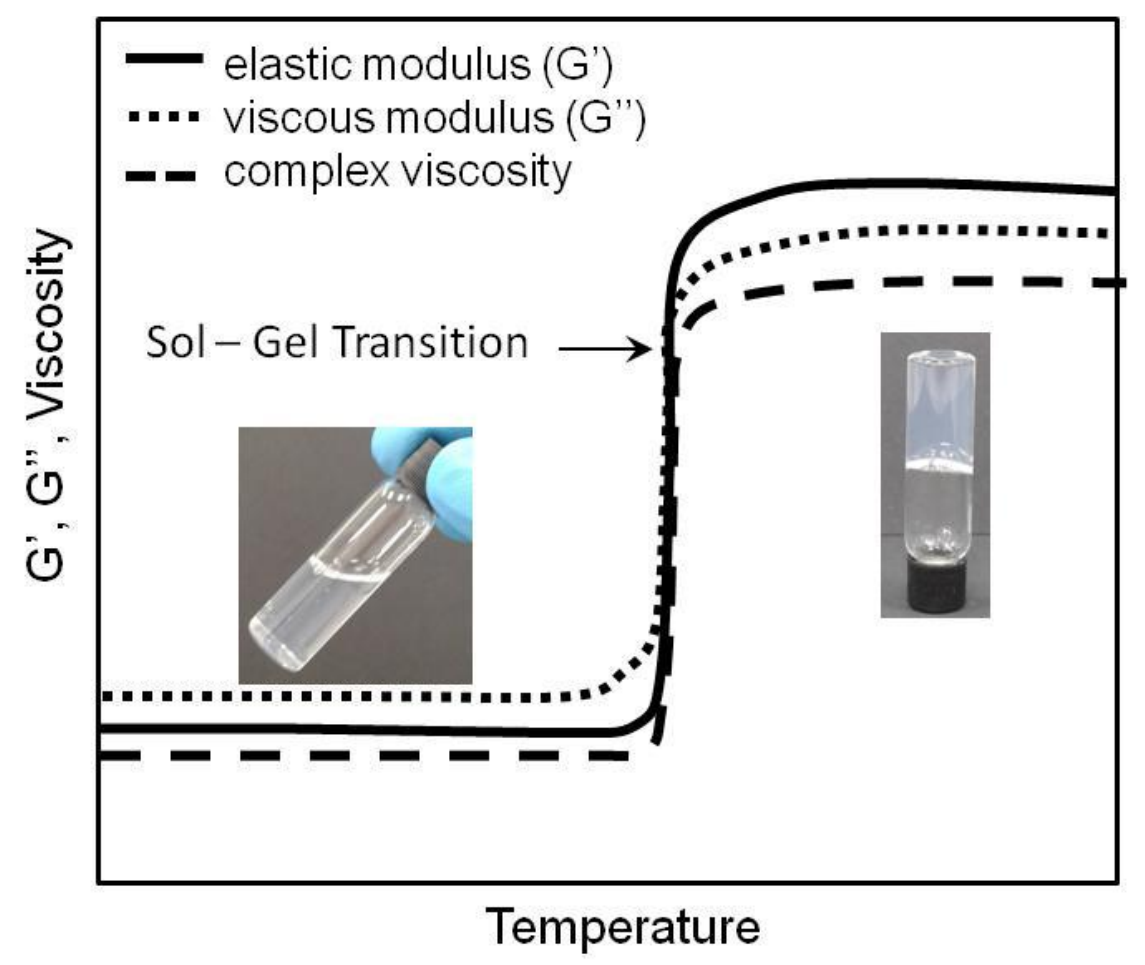

Figure 3. Sol-gel transition as it is monitored by rheology. G' (elastic modulus), G". (viscous modulus) and complex viscosity are shown in solid, dotted and dashed lines, respectively. The gel point is defined as the temperature when elastic modulus, $\mathrm{G}^{\prime}$ exceeds the viscous modulus $\mathrm{G}^{\prime \prime}$. 


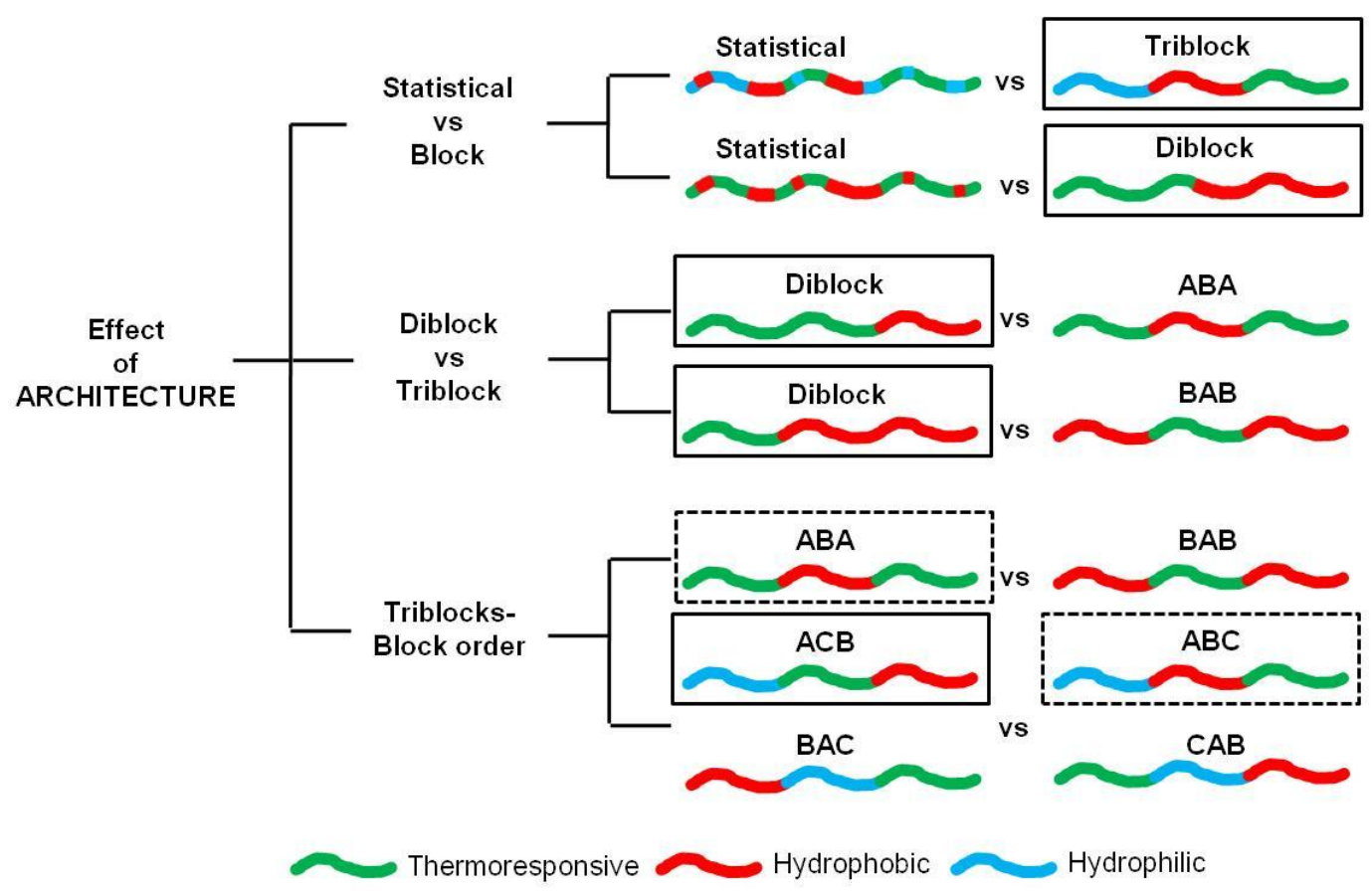

Figure 4. Schematic representations of the different polymers architectures. The thermoresponsive, hydrophilic and hydrophobic components are coloured in green, blue and red, respectively. With squares we emphasize which polymer structure forms gel easier. With dotted squares some discrepancies or interesting gel formations are noted. 
Table 1: Main Polymeric Characteristics that have been investigated and how these influence the formation of physical gels

\begin{tabular}{|c|c|c|}
\hline Polymeric Parameter that was varied & Trend/Effect on Gelation & References \\
\hline Molar Mass & $\begin{array}{l}\text { By increasing the MM the } T_{\text {gel }} \text { and CGC decrease, however } \\
\text { too high MMs often lead to precipitation/ }\end{array}$ & $37-42$ \\
\hline Composition; Hydrophilicity/Hydrophobicity & $\begin{array}{l}\text { By decreasing hydrophilicity (increasing hydrophobicity) the } \\
\qquad T_{\text {gel }} \text { and CGC decrease }\end{array}$ & $40,42,45-56$ \\
\hline Stereochemistry & No clear trend & $49,57,58$ \\
\hline Architecture: Random versus block structure & $\begin{array}{l}\text { Random polymers either do not form gels or form less stable } \\
\text { gels at higher } T_{\text {gel }}\end{array}$ & $45,47,54,59-61$ \\
\hline Architecture: Diblock versus Triblock & Diblocks seem to form gels easier. & $63,64,66-68$ \\
\hline Architecture: $\mathrm{ABC}$ vs $\mathrm{ACB}$ vs $\mathrm{BAC}$ terpolymers & $\begin{array}{l}\text { When A: hydrophilic B: hydrophobic and C: } \\
\text { thermoresponsive: BAC based structure polymer form gels } \\
\text { either at all temperatures or at lower } T_{\text {gel }} \text { s compared to the } \\
\text { other two triblock copolymer architectures. The ABC } \\
\text { triblock architecture was identified to be the "best" } \\
\text { architecture because it was producing the most clear, sharp } \\
\text { sol-gel transition and the gels seemed to remain more stable } \\
\text { compared to the two other architectures. }\end{array}$ & 45,54 \\
\hline
\end{tabular}

